\title{
Title: Universe At Zero Second And The Creation Process Of The First Particle From The Absolute Void
}

\author{
Shivan Sirdy ${ }^{*}$ (Corresponding author) \\ Independent researcher, Kirkuk, Iraq \\ *Corresponding Author Email address: sheevan91@gmail.com
}

\begin{abstract}
In this study, we discuss the properties of absolute void space or the universe at zero seconds, and how these properties play a vital role in creating a mechanism in which the very first particle gets created simultaneously everywhere and we find the limit in which when the absolute void volume reaches will lead to the collapse that leads to the creation of the first particle. This discussion is made following to the elementary dimensions theory study that was peer-reviewed at the end of 2020 , everything in the universe is made from four elementary dimensions, these dimensions are the three spatial dimensions ( $X, Y$, and $Z$ ) and the Void resistant as the factor of change among the four, time itself wasn't considered as the fourth dimension, rather time corresponds to a factor of change, and during the research it was found out that the Void resistant is the factor of change in the Absolute Void space, where time is a hypothetical concept, that represents changes during certain events compared to a constant change rate event.

Therefore, time does exist, but as a factor of change, and as the Void resistant in the absolute void space, Time $=$ factor of change $=$ Void resistant.

In the study, the internal and external void resistant volume equivalent is found, External Void resistant $=3.2857602 * 10^{\wedge} 15^{*}$ mass. This equation is used to identify to amount of Free external void resistant created during nuclear fission and fusion:

Initial mass of the excited nucleuses $\rightarrow$ mass of the created new nucleuses $+3.2857602 * 10^{\wedge} 15^{*}$ the lost Mass. In elementary dimensions the energy created during nuclear reactions is equivalent to the free External void resistant created through nuclear reactions, and mass is equivalent to the internal Void resistant.
\end{abstract}

Keywords: elementary dimensions; absolute void; time alternative; early universe; universe at zero second; Void resistant; Hydrogen atom; Hadron field; Lepton field

\section{$\underline{\text { Introduction }}$}

How come we exist?

The above question must have been the oldest one in the human mind and the most important one, or at least, that is how that question was to the author.

Twelve years ago, the author was hoping to find the answers through the work of the scientists and philosophers, since there have been smart minds throughout the history of mankind. Indeed, they contributed to countless breakthroughs in different sectors of science, 
Sociology...

They had developed some theories on where we came from. The most popular one must have been the big bang theory (Adams, Fred C.; Laughlin, Gregory (April 1997)). The theory states that about 13.7 billion years ago all the matter in the Universe was concentrated into a single incredibly tiny point. This began to enlarge rapidly in a hot explosion, and it is still expanding. A proof for the theory is that all other galaxies are moving away from us.

Unfortunately, the big bang or any other theory about the origin of the universe never explain how nothing did become something, or how do we exist? This forced the author to start his own journey to answer the question.

The goal of the paper is to provide an alternate answer to the questions about the origin of the universe

The scientific contributions of the paper are:

1. Introduce the Void resistant as a replacement to a factor of change, and as a Time in the absolute void space.

Time $=$ factor of change $=$ Void resistant

2. Present the limit of the level of instability in the Absolute Void space.

3. Provide an alternate solution to the origin of matter.

4. Introduce the hadron and lepton field as internal and external Void resistants

5. Introduce External void resistant equation: External Void resistant $=3.2857602 * 10^{\wedge} 15$ *mass. 


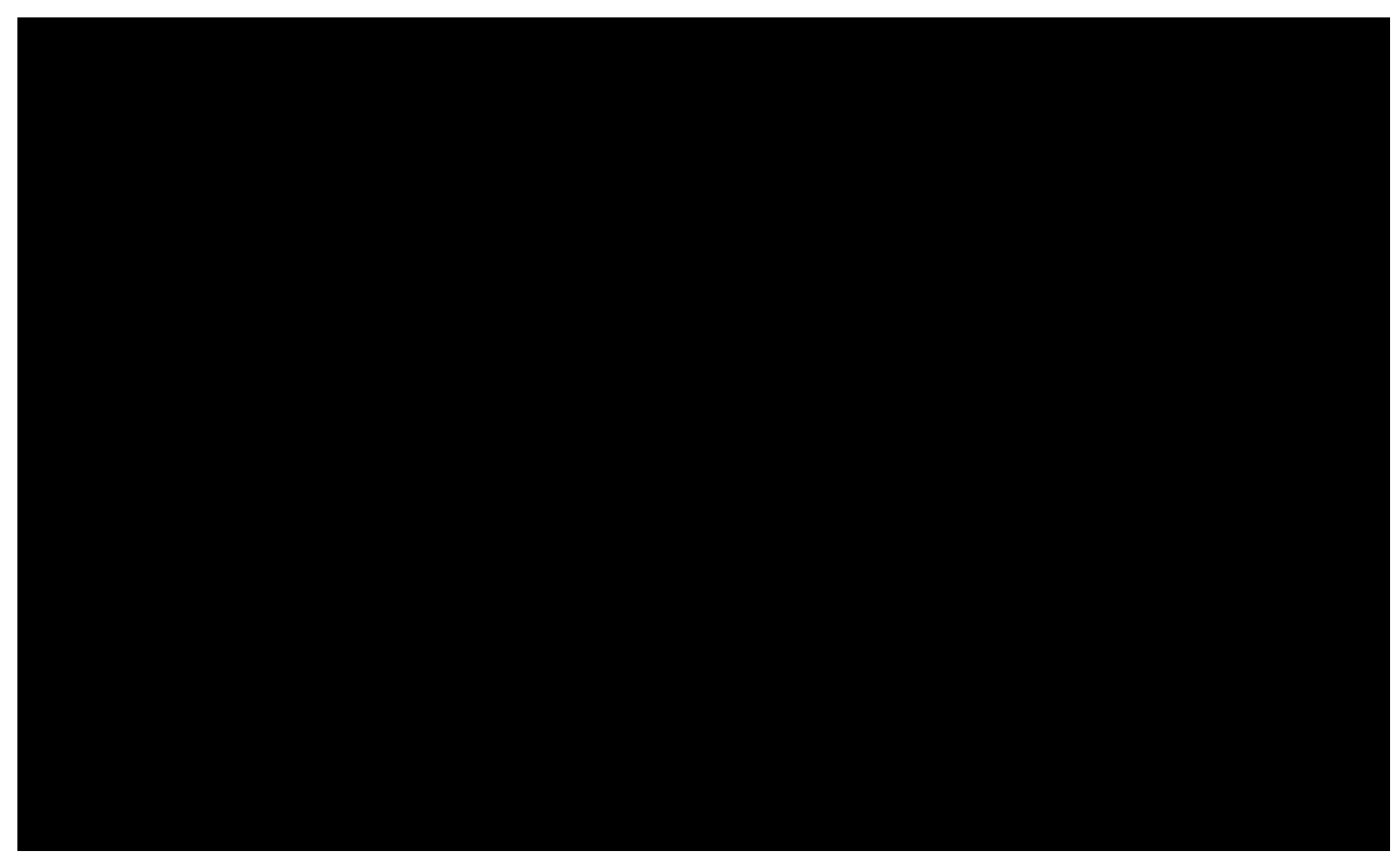

Figure 1(universe at time $=0$ sec or absolute void)

Universe at zero second (as shown in figure 1), the space was completely devoid of all sorts of particles and radiations, the absolute zero temperature was reached naturally, due to the absence of all sources of heat. This space is called absolute void (Sirdy, 2020).

Creating absolute void in the laboratory is quite challenging as the removal of all sorts of matter and radiation for the creation of absolute zero temperature is extremely difficult.

Absolute void is four-dimensional (space dimensions in the $x, y$, and $z$ axis and the Void resistant). The Void resistant is the factor of change among the four dimensions (Sirdy, 2020) which plays a vital factor in creating the first particle. Those four are the elementary dimensions. In the previous study the Void resistant was named Force field, however since it was creating confusion with the force known in physics it was changed to Void resistant.

Time is a hypothetical concept (Craig, William Lane, 2010), that corresponds to changes during certain events compared to a constant change rate event (Sirdy, 2020). Therefore, time itself wasn't considered as the fourth dimension. Though, time does exist but as a factor of change, and the Void resistant was identified as a factor of change in the absolute void space as it will be explained below. 
Time $=$ factor of change $=$ Void resistant

Therefore, every function that will be used in the EDs (the elementary dimensions) theory, are time invariant function (Oppenheim, Alan; Willsky, Alan (1997)).

This paper includes:

- Identification of the fourth dimension experiment: in his part we discuss the methodology that was used to identify the fourth dimension.

- Void Resistant patterns: In this part we discuss the patterns of the Void Resistant.

- Creation of the first particle through the elementary dimensions and the limit of the collapse: In this part we discuss the methodology where the first particle gets created simultaneously everywhere in the space of Absolute Void, then we find the limit where the system collapses creating the first particle, then we find the external and internal Void resistant equivalent that governs the Radius of the lepton field in relation to the hadron field.

- The collapse of a neutron to a proton + Lepton: In this part, we discuss the process in which a neutron collapses to a proton and a lepton.

- Nuclear fusion and fission: in this section, we introduce the nuclear reactions using the internal and external void resistants.

\section{Methodology}

\section{Identification of the fourth dimension}

The Void resistant or the factor of change was identified through experiment, Owing to lack of resources, a $10 \mathrm{ml}$ syringe was used to identify the fourth elementary dimensions of the void. The syringe head was closed, and the bottom part was pulled by using weights to create the void in the syringe. (Shown in figure 2) 


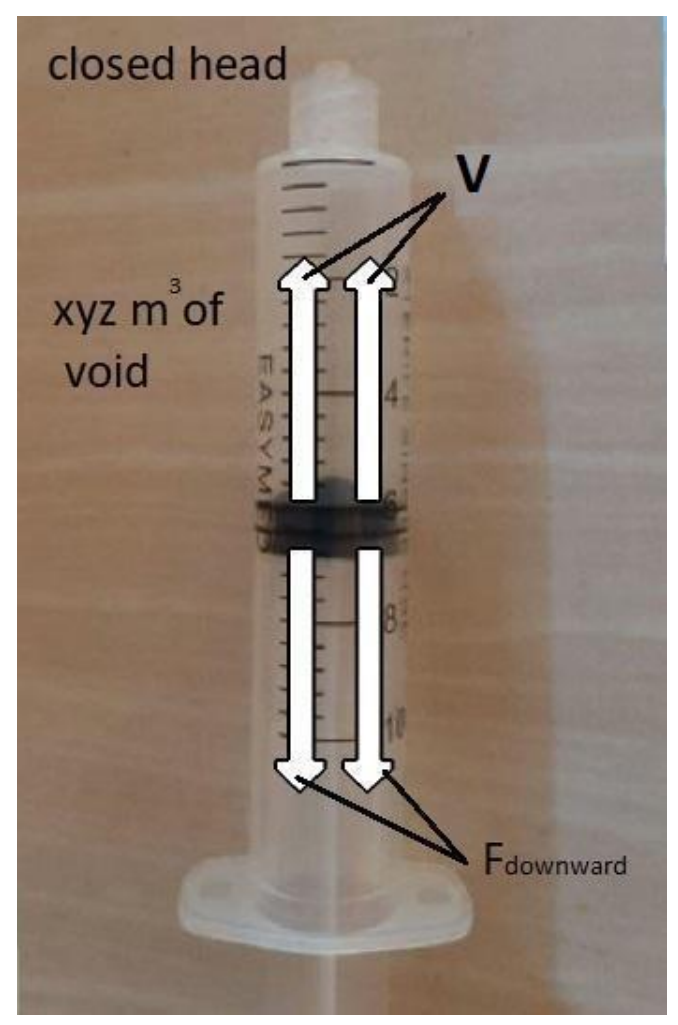

Figure 2 identification of the fourth dimension

The resistant force developed inside the syringe due to lack of matter was called Void resistant or just $\mathrm{V}$ for simplifications

Through classical physics, the summation of forces on the vertical axes equals zero

$\Sigma$ Fy=0, V+ Fdownward $=0$.

Where Fdownward is the force needed to pull the matter downward to create $x y z \mathrm{~m} 3$ of void, and $\mathrm{V}$ is the void resistant sourced from the void to resist the lack of matter.

$\mathrm{V}+$ Fdownward $=0, \mathrm{~V}=-$-Fdownward.

Therefore, the $\mathrm{V}$ or the void resistant corresponds to the factor of change

\section{Void resistant patterns}

The figure 3 and 4 shows the Void resistant pattern direction of an absolute void in a confined and an open system, respectively. 


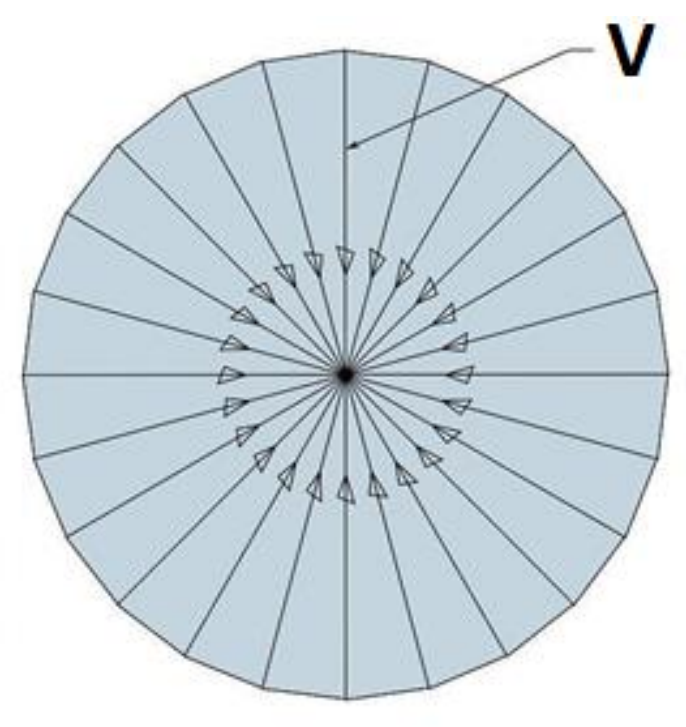

Figure 3 Void resistant patterns in a confined system

In Figure 3 the Void resistant acts to prevent the formation of the void by trying to crush the parameter of the surroundings toward the centre; the direction of $V$ is pointing toward the centre of the void. 


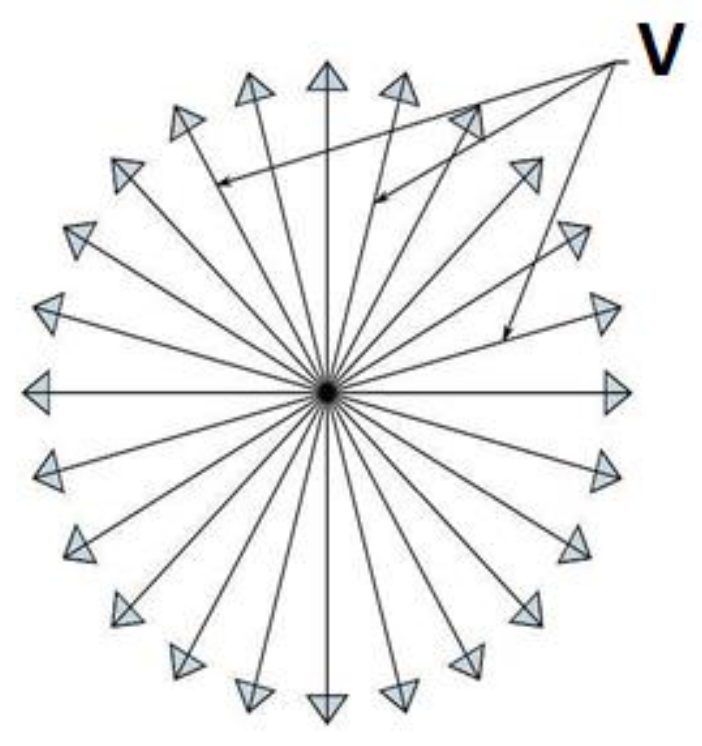

Figure 4 Void resistant pattern in an open system

However, absolute void is considered an open system, if it exists in a free form. At any point of it the Void resistant is pointing outward, as depicted in Figure 4.

Any point of absolute in a confined system acts as absolute void in an open system, which means that Void resistant is pointing outwards (see Figure 5).
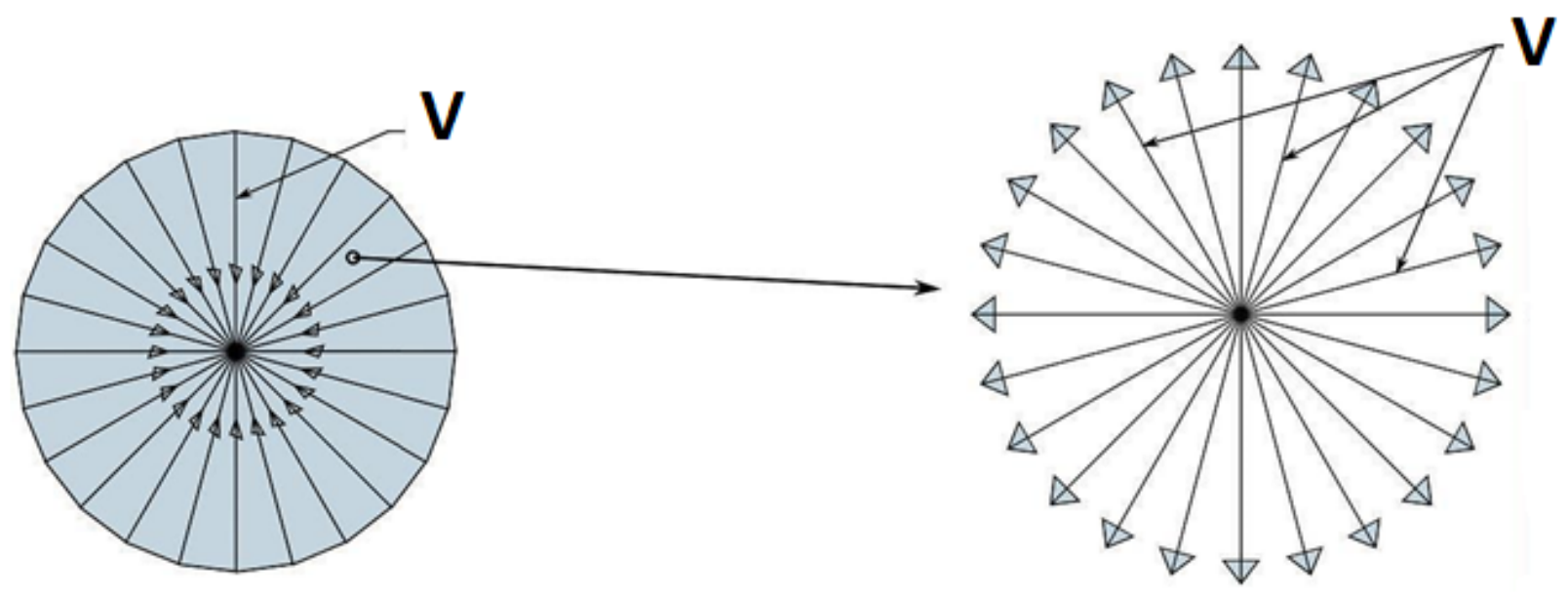

Figure 5 every point inside a confined system acts as an open system 
The Void resistant $(\mathrm{V})$ has a positive relation with the spatial dimensions ( $\mathrm{X}, \mathrm{Y}$ and $\mathrm{Z}$ ), meaning the Void resistant increases with the increase of the absolute void's volume $\mathrm{V} v$.

$\mathrm{V} \alpha \mathrm{Vv}$

$\mathrm{V}=\mathrm{Cv} * \mathrm{Vv}$

Where $\mathrm{Cv}$ is the void constant that was assumed, here we will again assume that it's equal to 1 with SI base units of Kg.m-2.sec-2

The volume of sphere $V v=\frac{4}{3} \pi(R 3)$

$V=\frac{4}{3} \pi(R 3) C V$

V will have the units of force $N$ (Newton), excluding the time effect completely, as this will be as well a time invariant function.

\section{Creation of the first particle through the elementary dimensions}

According to the elementary dimensions EDs theory, the absolute void and its four EDs are the predecessor of particles (Sirdy, 2020), and the EDs makes up everything in the universe. Contrarily to the current theories, according to the EDs theory a dot particle (or a one dimensional particle) is impossible to exist due to the mathematical impossibility and the absence of an existing actual physical limit determining the smallest mathematical and physical size. Therefore, every particle no matter how tiny it's, it must be having the three spatial dimensions ( $X, Y$, and $Z$ ) and according to the EDs theory it also has the fourth dimension (the factor of change or the Void resistant). 


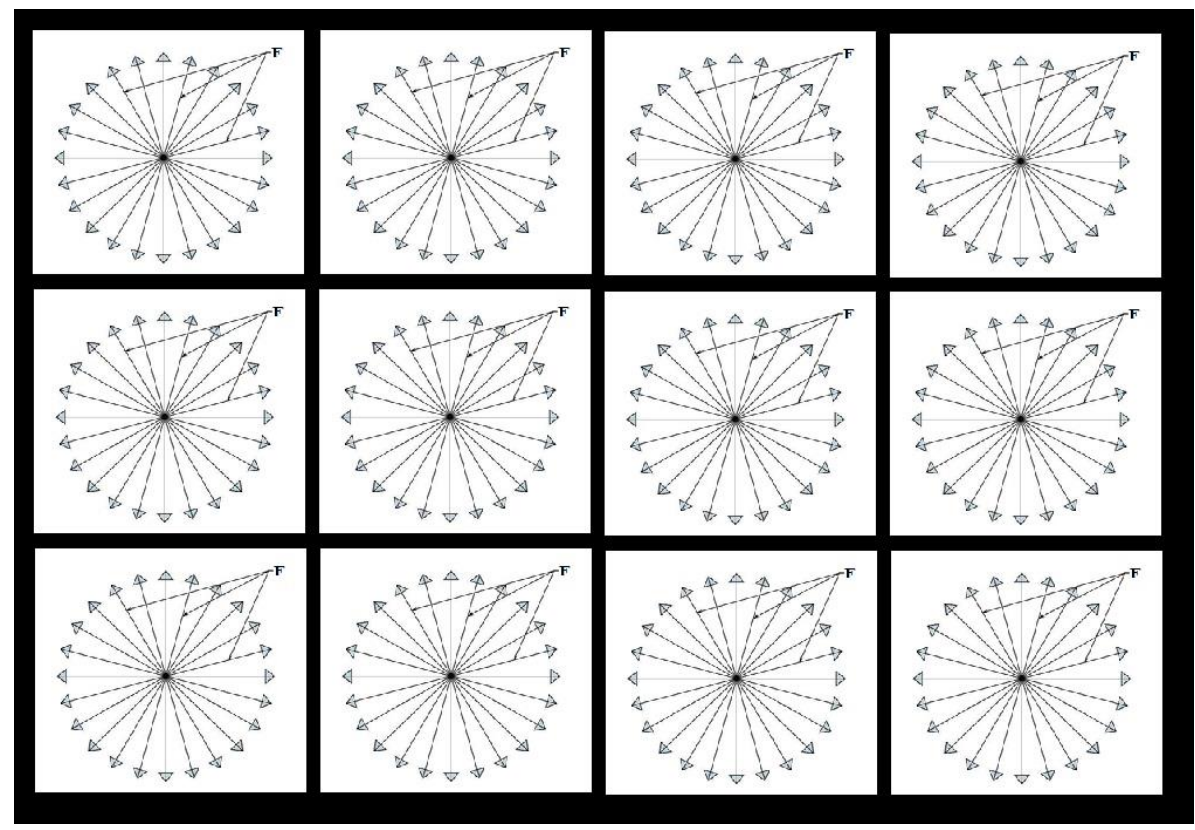

Figure 6 Universe at zero second with radius of $X$

Figure 6 visualizes a cross section of the universe at zero second and how it's only made of the outward Void resistant, as the system is an open system.

With the increase of the absolute void's volume, the outward Void resistant increases, and the space losses equilibrium more and more due to the presence of only one directional Void resistant. When the outward Void resistant reaches a certain limit (the limit will be discussed below), the system reaches the highest level of instability. The outward Void resistant has to collapse inward to restore equilibrium in the space (see Figure 7).
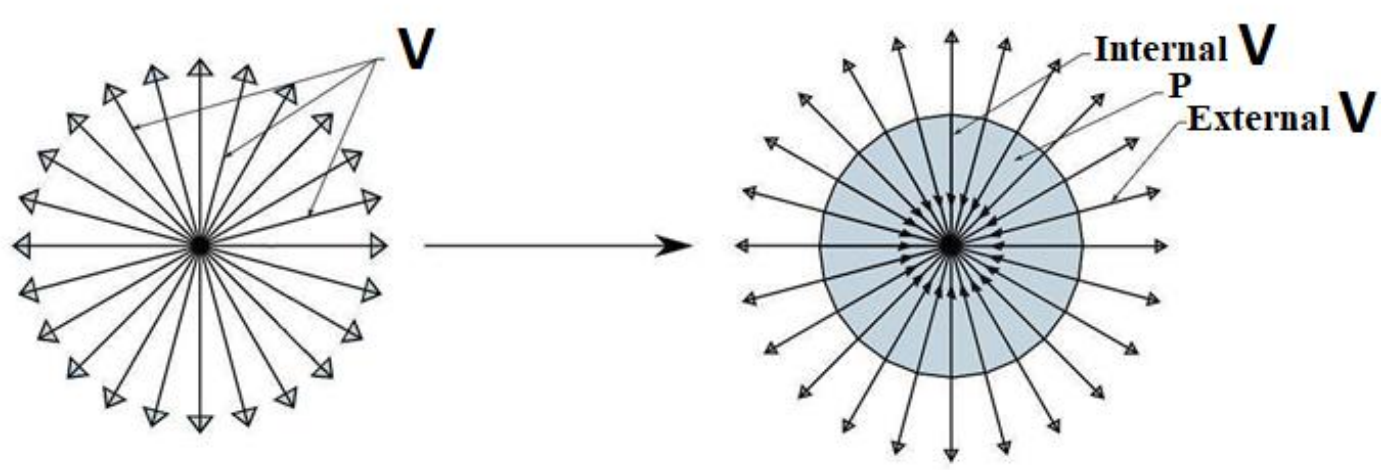

Figure 7 the change from an open system to a confined system 
The absolute void has turned from an open to a confined system, with the collapse energy was created from the Void resistant pointed toward the centre, the energy stays conserved at all phases. However, due to the collapse the energy density increases with the decrease of the spatial dimensions (see figure 8).

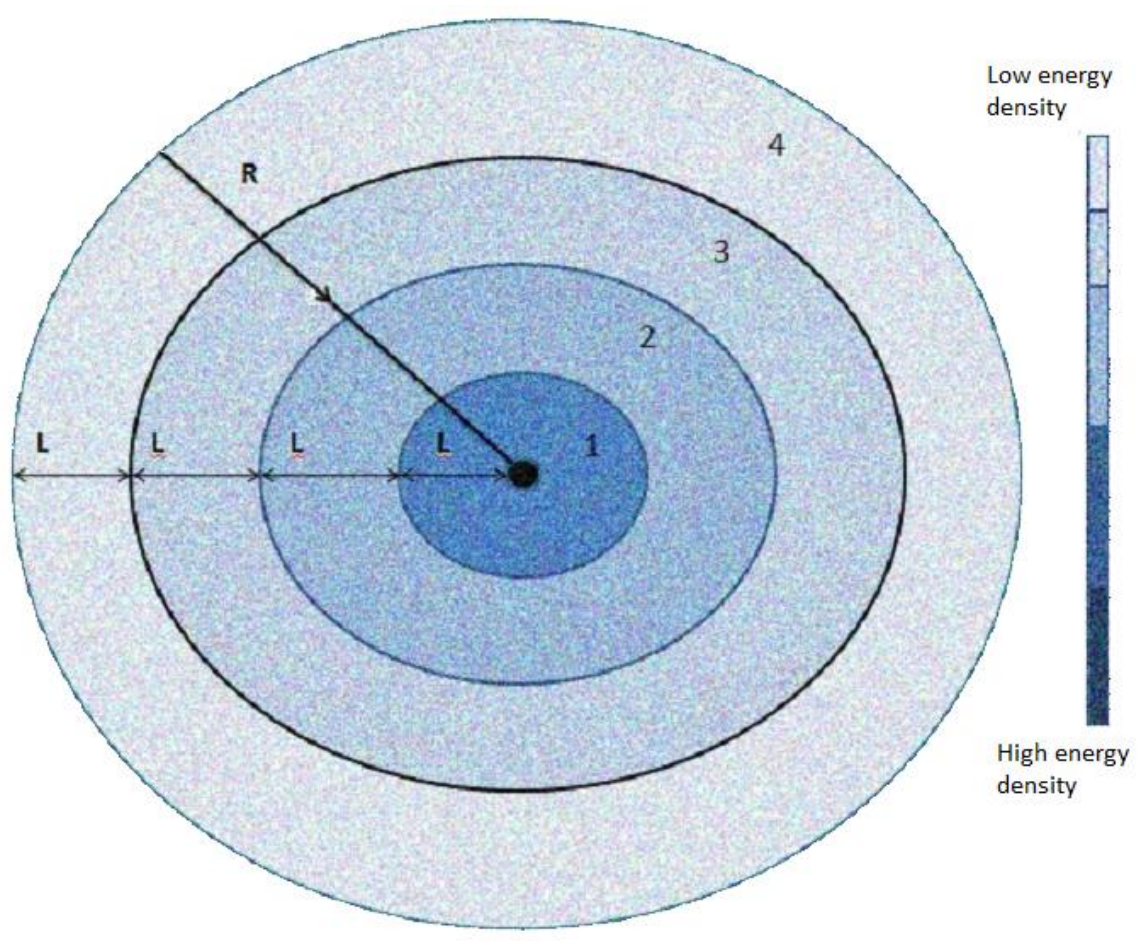

Figure 8 increase of the energy density with the collapse

At the final stage the energy is concentrated forming a solid shape of energy. In which, this shape of energy is a first particle that was ever created from the EDs. This particle was created to decrease the instability in the space to achieve equilibrium that the universe at time zero second was lacking.

This particle is managed by two forces:

1. Internal Void resistant $\mathrm{Vi}$, which originates from the absolute void inside the particle, just like void in a confined system, and is directed toward the centre.

2. External Void resistant Ve, originates from the absolute void outside the created particle.

The Void resistant Vi causes the source to collapse, while the Void resistant Ve is the counter factor. The particle collapses until equilibrium is achieved between the internal and external Void resistant. In case, more than one source-like entity are existents near each other relatively, the equilibrium process is among all of them. 
At time zero second, infinite spatial dimensions of absolute void existed. Therefore, unlimited numbers of particles were created (see figure 9).

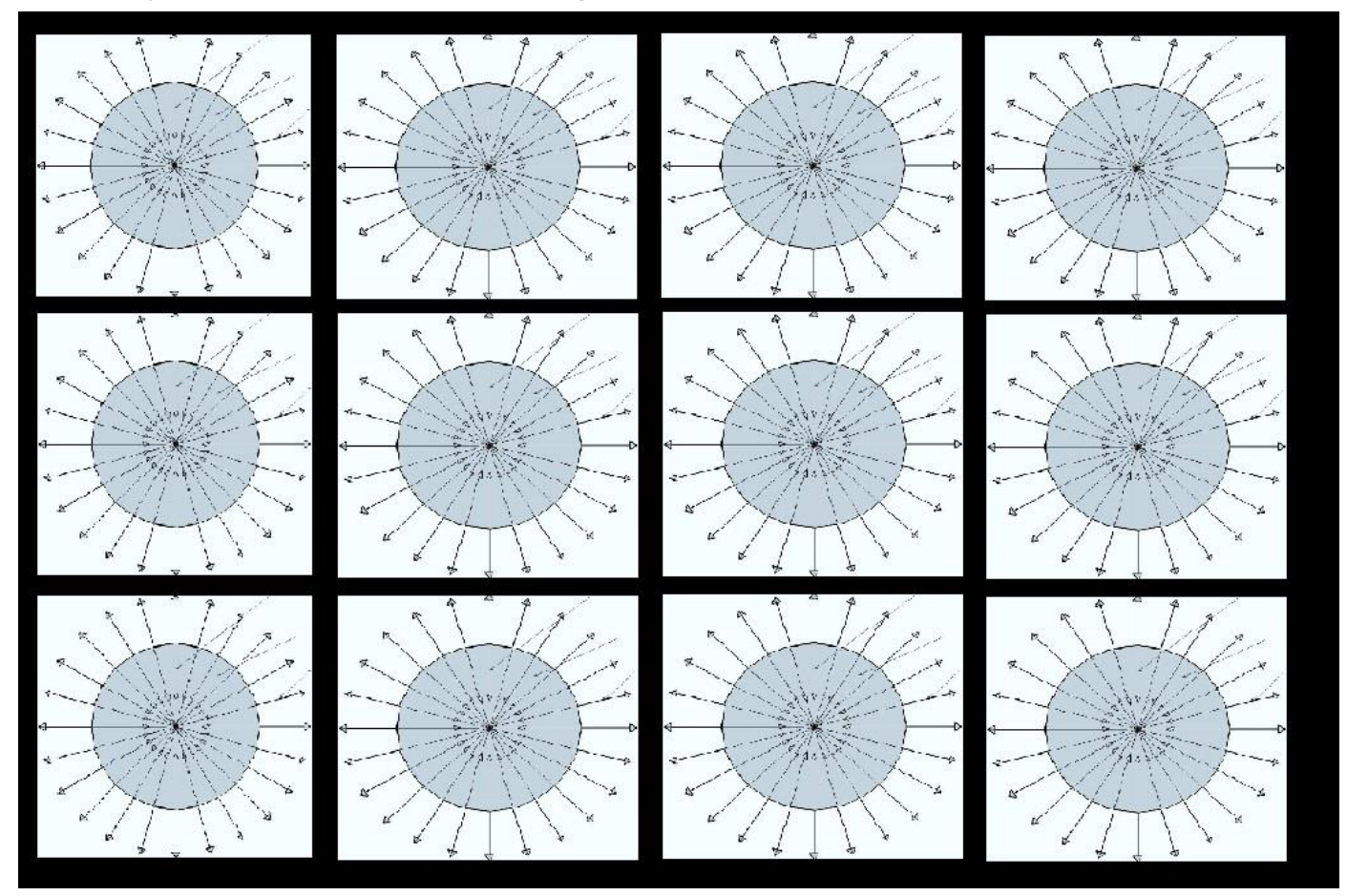

Figure 9 (infinity numbers of particles created from the EDs)

Figure 9 visualizes a cross section of the universe after the collapse and how the system has turned from an open system to a confined system.

The limit in which the system collapses might be the same limit that leads to the creation of the

black holes, where it's already know that for large stars above the

Landau/Tolman-Oppenheimer-Volkoff limit (Wheeler, J. C.; Grossan, B; Pooley, D.; Kumar, P.;

(2018-05-31)) (around two solar masses), when the star dies the forms of particles cannot provide

the force needed to balance gravitational field. There is nothing to stop the collapse forming a black

hole (Pooley, D.; Kumar, P.; Wheeler, J. C.; Grossan, B. (2018-05-31).

A black hole is similar to the origin particle or the source (Sirdy, 2020).

The limit could be a radius of $8.7713 \times 10^{8}$ meters of absolute void in a sphere shape, using a value 
of $3.98 \times 1030 \mathrm{Kg}$ (two solar masses) with density of $1408(\mathrm{~kg} / \mathrm{m} 3)$, the same density of our sun.

Using mass density equation:

Denisity $=\frac{\text { two solar masses }}{\text { Volume of the star }}$

$1408=\frac{3.98 \times 10^{\wedge} 30 \mathrm{Kg}}{\frac{4}{3} \pi\left(\mathrm{R}^{\wedge} 3\right)}$

We get the value of a radius $8.7713 \times 108$ meters of absolute void,

Using the Void resistant equation (6):

$V=\frac{4}{3} \pi(R 3) C v$

$V=2.82671 \times 10^{\wedge} 27 \mathrm{~N}$

Meaning when the absolute void's Void resistant reaches this value, the space inside the sphere will be at its highest level of instability, the only way for it to achieve equilibrium is to collapse inward.

The spatial dimensions of the two solar masses its radius equivales to its Schwarzschild radius,

Meaning the open system radius is equivalent to its closed system radius

Radius of two solar masses=Its Schwarzschild radius* equivalent.

$8.7713 \times 10^{\wedge} 8=5.9 * 10^{\wedge} 3^{*}$ Radius equivalent

Radius Equivalent=148666.1

Meaning:

Open system radius $=148666.1$ closed system radius

Open system is the external void resistant

Closed system is the internal void resistant 
Of course, the same thing can be said to their volume, which means that we also have Volume equivalent:

External void resistant Volume $=$ Internal void resistant Volume $*$ Volume equivalent.....(10)

$4 / 3 * \mathrm{Pi}^{*}\left(8.7713 \times 10^{\wedge} 8\right)^{\wedge} 3=4 / 3^{*} \mathrm{Pi}^{*}\left(5.9^{*} 10^{\wedge} 3\right)^{\wedge} 3^{*}$ Volume equivalent

Volume equivalent $=3.2857602 * 10^{15}$

External Void resistant $=3.2857602 * 10^{15} *$ Internal Void resistant

Hydrogen radius

Using radius equivalent

Atom radius $=148666.1 *$ Proton radius (12)

$=148666.1 * 0.85^{*} 10^{\wedge}-15=200.5422^{*} 10^{\wedge}-12$ meter $=126.3662$ picometer
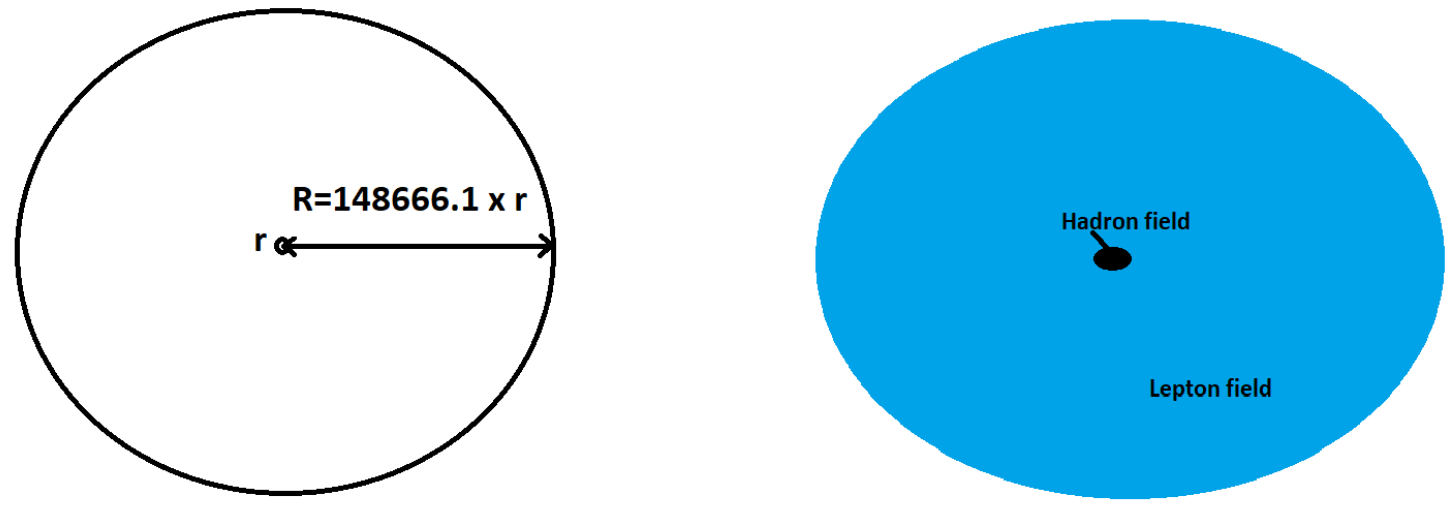

Figure 10 Hydrogen atom sample analysed using the external and internal Void resistant ratios 


\section{The collapse of a neutron to a proton + Lepton}

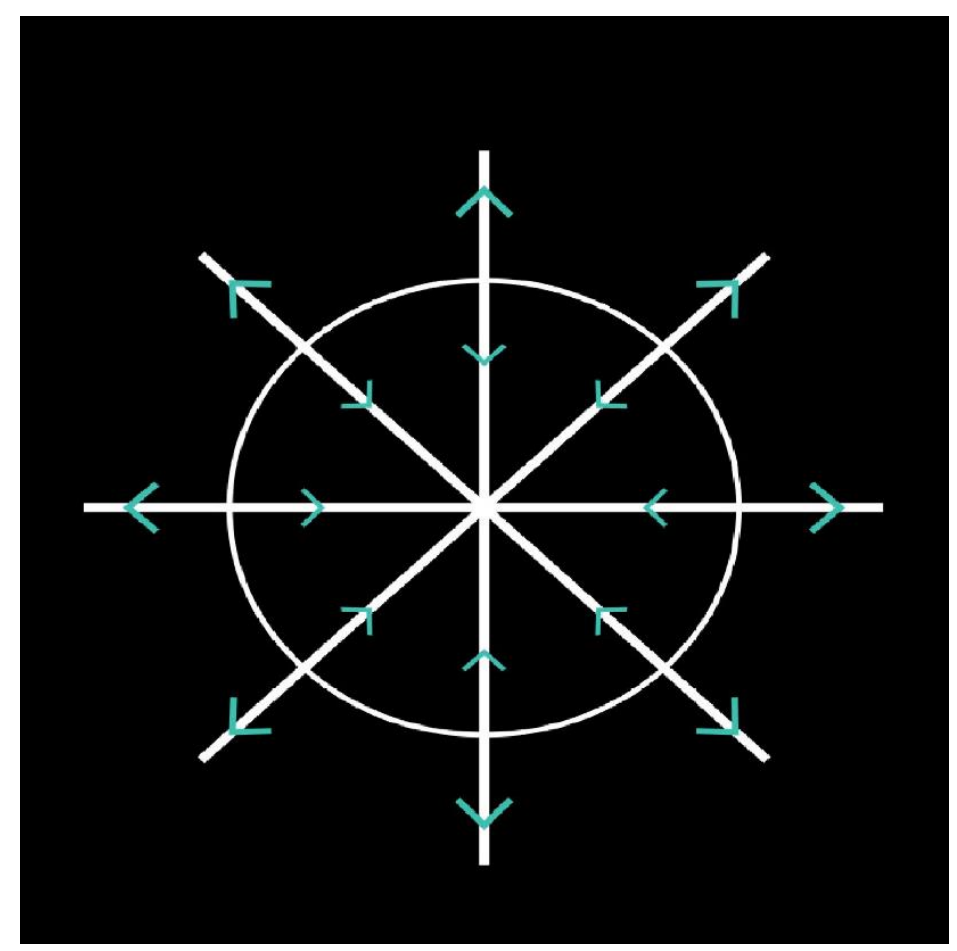

Figure 11 Neutron

The above Figure 11 shows a neutron before it collapses, as a neutron is a particle governed by the Internal Void resistant that tends to make the particle collapsing and the External Void resistant that tends to stop the collapse. In a neutron, the Internal Void resistant is greater than the internal Void resistant, therefore the external Void resistant is not enough to stop the particle from collapsing. 


\section{Hydrogen atom}

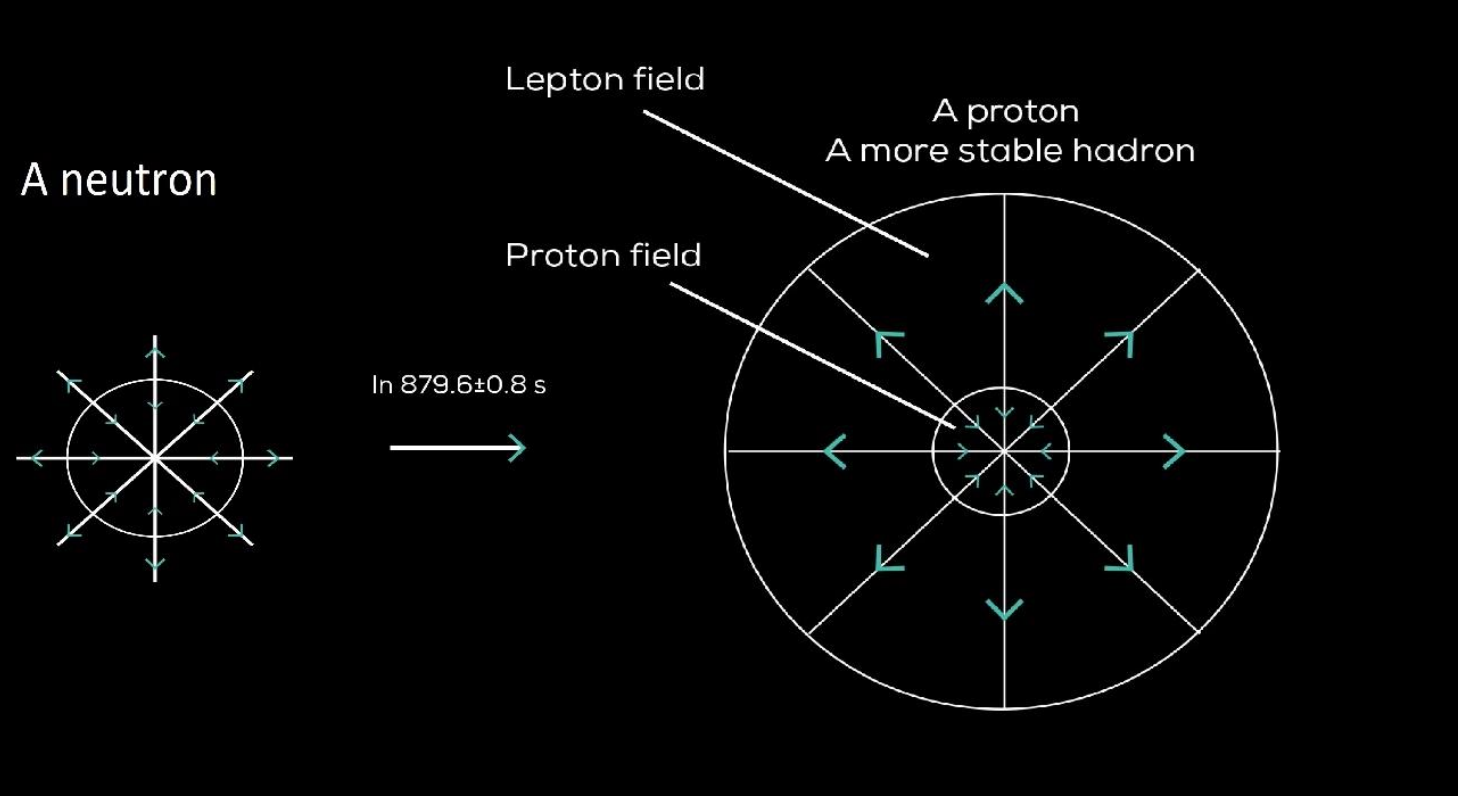

Figure 12 the collapse of a neutron to a proton+Lepton

In figure 12, in 879.6 plus minus 0.8 seconds a neutron has collapsed due to the effect of the Internal Void resistant being larger than the External Void resistant, and when both of the resistants are equal to each other, the system of the particle becomes in equilibrium, The Internal Void resistant will be called a proton field, and the External Void resistant will be called the Lepton field. Whenever, a system collapses, electro-magnetic field gets created from the effect of the External Void resistant on the system as a free field spreading outward (see figure 13,14, and 15).

As we can see above in the figures that the internal void resistant is equivalent to mass Mass=Internal void resistant

Returning the equation (11):

External Void resistant $=3.2857602 * 1015 *$ Internal Void resistant

The internal void resistant can substituted with mass,

External Void resistant $=3.2857602 * 10^{\wedge} 15 *$ Mass. 


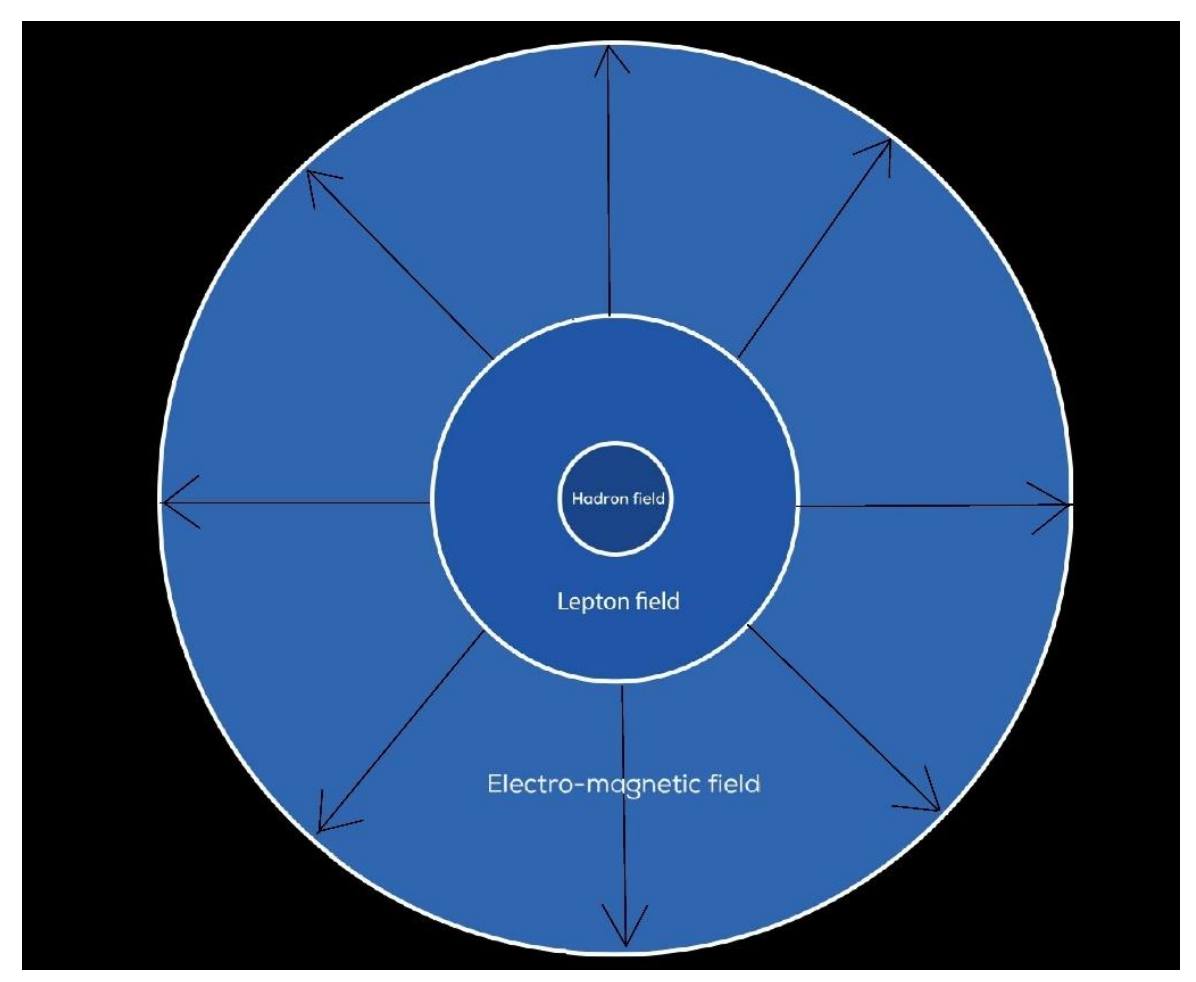

Figure 13 The three main fields

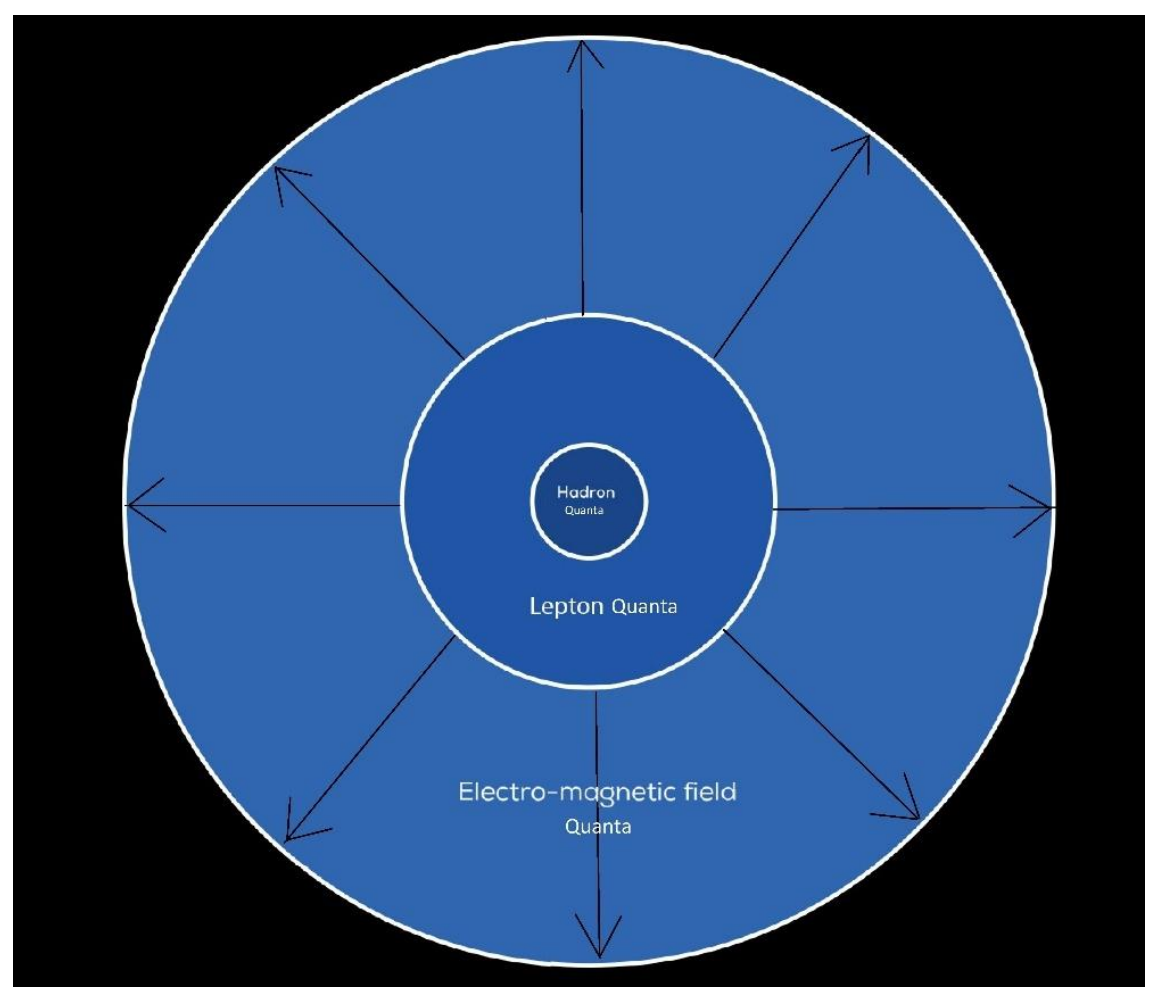

Figure 14 each of the three fields can be quantized 


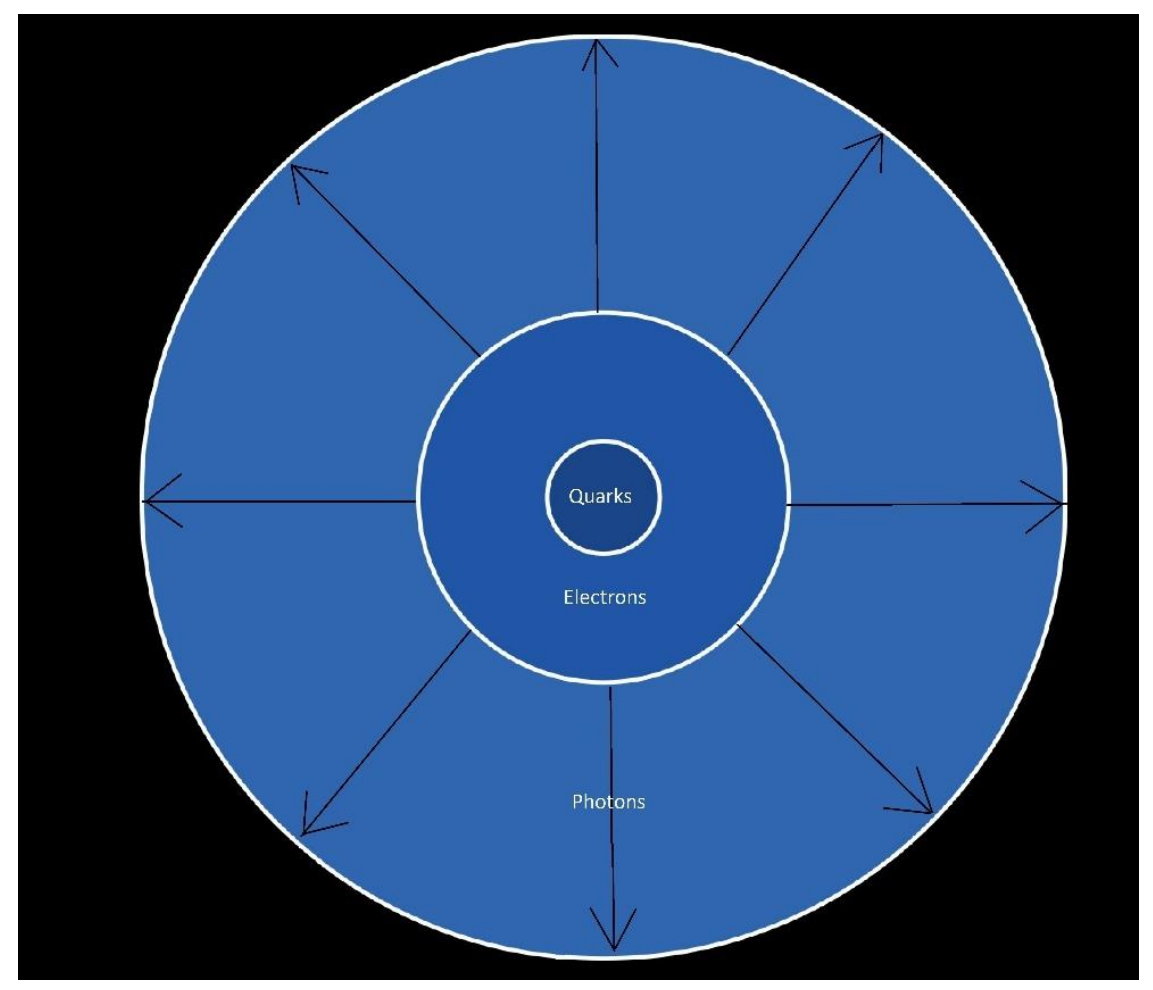

Figure 15 Quanta of each of the three main fields

\section{Nuclear fusion and fission}

Nuclear Fission is the process of splitting of a heavy, unstable nucleus into two lighter nuclei, and fusion is where two light nuclei combine releasing an amounts of energy (Conn, Robert W (2019)), or something else.

It's known that the nuclear fusion and fission can be expressed as the below formula:

Initial mass of the excited nucleuses $\rightarrow$ Mass of the created new nucleuses + Energy

According to the EDs theory, mass is equivalent to the internal void resistant, while energy is not defined yet. There is only one way for energy concept to fit in the EDs theory, and that is the fact that energy is the free external void resistant,

Free External Void resistant=Energy

Now we return to formula (15), but this time we use the EDs fundamental properties:

Initial Internal Void resistant of the excited nucleuses $\rightarrow$ Internal Void resistant of the created new nucleuses+ External Void resistant

And since External void resistant $=3.2857602 * 10^{\wedge} 15 *$ Mass (equation 14 ),

Initial Internal Void resistant of the excited nucleuses $\rightarrow$ Internal Void resistant of the created new nucleuses $+3.2857602 * 10^{\wedge} 15 *$ the lost Mass

We can put the above formula in a calculable shape through:

Initial mass of the excited nucleuses $\rightarrow$ mass of the created new nucleuses $+3.2857602 * 10^{\wedge} 15 *$ the lost Mass 
$3.2857602 * 10^{\wedge} 15 *$ the lost mass represents the amount of the free external void resistant created through the process of the nuclear fusion or fission.

Now through the above formula we are able to accurately calculate the amount of change that occurs in a system during nuclear fusion or fission.

Taking Deuterium- Deuterium fusion as an example:

${ }_{1}^{2} \mathrm{H}+{ }_{1}^{2} \mathrm{H} \rightarrow{ }_{2}^{3} \mathrm{He}+{ }_{0}^{1} n+3.27 \mathrm{MeV}$

${ }_{P}^{A} N$, where $\mathrm{N}$ is nucleus, A number of protons plus neutrons, $\mathrm{P}$ is number of protons, and $\mathrm{n}$ is neutron

Now shifting to the EDs principles:

${ }_{1}^{2} \mathrm{H}+{ }_{1}^{2} \mathrm{H} \rightarrow{ }_{2}^{3} \mathrm{He}+{ }_{0}^{1} n+$ Lost mass $* 3.2857602 * 10^{\wedge} 15$

And the same applies to every other fusion or fission.

\section{Conclusion}

In the universe at zero second only absolute void existed, absolute void is made from EDs., three spatial dimensions ( $X$ axis, $Y$ axis and $Z$ axis) and the Void resistant. When the sphere volume of absolute void reaches a radius of $8.7713 \times 10^{8}$ meters, the space inside the sphere will be at its highest level of instability, the only way for it to achieve equilibrium is to collapse inward, creating the first particle.

Universe at zero second, infinity spatial dimensions of absolute void existed. Therefore, unlimited numbers of particles were created simultaneously everywhere.

External Void resistant $=3.2857602 * 10^{\wedge} 15 *$ mass, this equation can be used whenever there is a release in the External void resistant or energy as a result of Internal void resistant conversion .

\section{Acknowledgments}

I would like to express my gratitude to Salahdin university and specifically (Dr Ameer)- Physics department for providing comments and feedback about the current study.

\section{REFERENCES}

1. Adams, Fred C.; Laughlin, Gregory (April 1997). "A dying universe: the long-term fate and evolution of astrophysical objects". Reviews of Modern Physics. 69 (2): 337-372. arXiv:astro-ph/9701131. Bibcode:1997RvMP...69..337A. doi:10.1103/RevModPhys.69.337. S2CID 12173790.

2. Craig, William Lane, the Tenseless Theory of Time, Springer, 2010.

3. Sirdy, S. (2020, December 31). The Role of Elementary Dimensions in the Creation of the Source of Elementary Particles. UKH Journal of Science and Engineering, 4(2), 69-83. https://doi.org/https://doi.org/10.25079/ukhjse.v4n2y2020.pp69-83

4. Oppenheim, Alan; Willsky, Alan (1997). Signals and Systems (second ed.). Prentice Hall.

5. Pooley, D.; Kumar, P.; Wheeler, J. C.; Grossan, B. (2018-05-31). "GW170817 Most Likely Made a Black Hole". The Astrophysical Journal. 859 (2): L23. arXiv:1712.03240. Bibcode:2018 ApJ...859L..23P. doi:10.3847/2041-8213/aac3d6.

6. Conn, Robert W.. "Nuclear fusion". Encyclopedia Britannica, 5 Jul. 2019, https://www.britannica.com/science/nuclear-fusion. Accessed 23 August 2021. 